\title{
CONFIGURACIONES DE LA FORMACIÓN DOCENTE INICIAL EN ARGENTINA (2015-2019): DE LAS COMPETENCIAS A LA FABRICACIÓN DE RESULTADOS
}

\author{
Raúl Enrique Piazzentino* \\ Escuela Normal Superior “Martiniano Leguizamón”, Argentina \\ rpiazzentino@hotmail.com
}

Recibido: 28/06/2020 - Aceptado: 10/11/2020

\section{Resumen}

Avanzada la primera década de los años dos mil hasta la actualidad, las políticas de formación docente en Argentina se configuran mediante la modalidad discursiva de Planes Nacionales de Formación Docente, representando una mudanza con relación a la formulación de planes de estudio y programas de formación de las últimas décadas del siglo XX.

En esta producción analizamos desde una perspectiva político discursiva, en línea con la Teoría de Discurso y el Análisis Político de Discurso, el Plan Nacional de Formación Docente 20152019 , entre otros documentos y normativas oficiales del período. Este conjunto de textos se halla imbricado en el discurso de las políticas para la formación docente inicial, organizado en torno al significante privilegiado de las competencias profesionales en el currículo.

A partir del significante privilegiado, reconocemos el delineado de un dispositivo de fabricación de resultados que procura, por una parte, dar cuenta de una ineficiencia de la formación docente inicial vigente y, por otra, orienta la mejora de la eficiencia hacia la fijación de estándares basados en competencias profesionales. Desde los productos de este dispositivo podemos reconocer las trazas de un modelo de identificación desde el que se interpela a las distintas posiciones del sistema formador, en línea con tendencias de signo neoliberal de estandarización y eficientismo impuestas a nivel regional desde países hegemónicos.

Palabras clave: Formación Docente Inicial - Planes Nacionales de Argentina - Competencias Profesionales - Estandarización - Análisis Político de Discurso.

\section{CONFIGURATIONS OF INITIAL TEACHER TRAINING IN ARGENTINA (2015-2019): FROM COMPETENCES TO RESULTS MANUFACTURING}

\begin{abstract}
Advanced the first decade of the two thousand years to the present, teacher training policies in Argentina are configured through the discursive modality of National Teacher Training Plans, representing a change in relation to the formulation of study plans and training programs of the last decades of the 20th century.

In this production we analyze from a political discursive perspective, in line with the Theory of Discourse and Political Analysis of Discourse, the National Plan for Teacher Training 2015-2019, among other documents and official regulations of the period. This set of texts is embedded in the
\end{abstract}

\footnotetext{
* Doctor en Educación, Facultad de Ciencias de la Educación, Universidad Nacional de Entre Ríos. Actualmente desempeña la docencia y la rectoría en la Escuela Normal Superior "Martiniano Leguizamón" de Villaguay, Entre Ríos.
} 
discourse of policies for initial teacher training, organized around the privileged signifier of professional competencies in the curriculum.

Starting from the privileged signifier, we recognize the outlining of a device for manufacturing results that seeks, on the one hand, to account for an inefficiency of the current initial teacher training and, on the other, guides the improvement of efficiency towards the setting of standards based on professional competencies. From the products of this device we can recognize the traces of an identification model from which the different positions of the training system are challenged, in line with the neoliberal trends of standardization and efficiency imposed at the regional level from hegemonic countries.

Keywords: Initial Teacher Training - National Plans of Argentina - Professional Competencies Standardization - Political Analysis of Discourse.

\section{Introducción}

Avanzada la primera década de los años dos mil hasta la actualidad, las políticas de formación docente en Argentina se configuran mediante la modalidad discursiva de Planes Nacionales de Formación Docente (PNFD), representando una mudanza con relación a la formulación de planes de estudio y programas de formación de las últimas décadas del siglo XX. A partir del análisis de las superficies de los textos de los PNFD se patentizan los puntos nodales que dan sentido a las configuraciones discursivas de las políticas educativas para la formación docente inicial en las que se inscriben. En ese sentido, en esta producción analizamos el PNFD 2015-2019 desde una perspectiva político discursiva, dando cuenta de las marcas del punto nodal de las competencias profesionales en el discurso de las políticas para la formación docente inicial.

El análisis de los documentos y normativas que expresan las políticas de formación docente del período delimitado, se inscribe en una perspectiva de indagación centrada en la dimensión de lo político, en línea con la Teoría de Discurso de Ernesto Laclau y Chantal Mouffe (1987), y los aportes de Rosa Buenfil Burgos (1992; 2019) desde el Análisis Político de Discurso (APD). En cuanto a la delimitación del corpus, partimos de un conjunto de textos de referencia (Howarth, 2005) a los que se incorporan otros, teniendo en cuenta las marcas reconocidas en las superficies textuales. En tal sentido, los textos resultan producciones en los que circulan y se diseminan los rasgos distintivos o regularidades de las configuraciones discursivas en las que se inscriben (Cruz Pineda, 2019) y pueden ser analizados como productos que admiten una reconstrucción de sus condiciones de producción (Verón, 2004).

El artículo está organizado en tres apartados, en el primero proporcionamos algunas trazas de una perspectiva político discursiva para la lectura de las políticas educativas, siguiendo los aportes de autores en la línea del APD. En la segunda sección consideramos, en líneas generales, las modalidades documentales de los cuatro PNFD aprobados desde 2007 al 2019 en el ámbito del Consejo Federal de Educación (CFE), con el propósito de señalar los puntos discursivos privilegiados en torno a los cuales se articulan los discursos de las políticas para la formación docente inicial. Finalmente, en la tercera sección, nos centramos, en particular, en el análisis del PNFD 2016 - 2019 (Resolución CFE N.ㅇ 286/16) y el Marco Referencial de Capacidades Profesionales de la Formación Docente Inicial (Resolución CFE N.․ 337/18), entre otros textos producidos durante el gobierno de la Alianza Cambiemos. Con ello nos proponemos identificar marcas vinculadas, por un lado, al punto discursivo privilegiado de las competencias profesionales en el currículo de la formación docente inicial, en el marco de operaciones discursivas tendencialmente hegemónicas a nivel regional y global. Por otro lado, a partir de las competencias en el currículo, dar cuenta del entramado de un dispositivo de fabricación de resultados que, a la vez, opera como modelo de identificación (Buenfil Burgos, 2009) desde el que se interpela a las distintas posiciones del sistema formador.

\section{Una aproximación a las políticas educativas desde la dimensión de lo político}

En esta primera sección del artículo recuperamos algunas aportaciones desde la analítica del APD, en línea con la Teoría de Discurso de Ernesto Laclau y Chantal Mouffe (1987), y su apropiación en el campo de la investigación educativa en Latinoamérica (Buenfil Burgos, 2019). El APD, en los términos de Buenfil Burgos (2011, p.102), resulta una perspectiva analítica posfundacional que procura entender e interpretar desde la dimensión constitutiva de lo político, la producción y transformación de configuraciones significantes que intentan fijar precariamente sentidos en lo social.

Desde este horizonte de intelección, sostenemos que las políticas educativas, en general, resultan configuraciones discursivas (Ruiz Muñoz, 2005). Estas configuraciones constituyen entramados de acciones y lenguaje (Wittgenstein, 1988) producidas a partir de procesos articulatorios en los que intervienen diferentes posiciones, organizando los discursos en torno a puntos discursivos privilegiados (Torfing, 1998) que fijan provisoriamente los sentidos.

El análisis político discursivo de las políticas educativas, que en nuestro trabajo se recorta en torno a las fijaciones de sentido de las articulaciones para la formación inicial de docentes en la historia reciente en Argentina (2015-2019), se asienta en el carácter constitutivo de lo político en lo educativo. Lo político como el momento de la decisión, de la elección entre lo que se incluye y lo que se excluye, constituye una perspectiva de indagación en el estudio de las políticas educativas. No sólo en lo que respecta a los momentos de diseño, implementación y resultados sino, como señala Treviño $(2018$, p.68), a procesos más 
sutiles, calculados o no, como la búsqueda de producir sujetos, influir sus formaciones identitarias y los procesos de subjetivación.

EI APD constituye una caja de herramientas analíticas (Buenfil Burgos, 2007), a partir de las cuales analizamos un conjunto de textos legales y documentales (Giovine y Suasnábar, 2012) imbricados en la configuración de la política educativa para la formación docente inicial del período en estudio. Estos documentos oficiales, delimitados en un corpus, constituyen nuestro referente empírico ${ }^{1}$, recortado inicialmente en un conjunto de textos de referencia (Howarth, 2005) a los que se incorporan otros, teniendo en cuenta las marcas que ofrecen las superficies textuales.

Siguiendo a Cruz Pineda (2008, p. 130), conceptualizamos y usamos las normativas y documentos oficiales como textos. En tal sentido, los reconocemos como resultantes de múltiples tejidos discursivos, en cuyas superficies textuales es posible indagar y analizar sobre las marcas e indicios que han hecho posible su conformación. En línea con las aproximaciones analíticas de la autora, recuperamos los señalamientos de Verón ${ }^{2}$ respecto al reconocimiento de marcas en los textos de referencia, concebidos en términos de huellas de operaciones discursivas, a partir de las cuales es posible reconstruir las condiciones de producción, constituidas por otros textos. Finalmente, como plantea el autor, un texto forma parte de las condiciones de producción si, y solo si, ha dejado marcas en los textos de referencia (1993, p.18).

\section{Algunos sentidos de los discursos de la formación docente inicial}

Las políticas de formación docente en las dos primeras décadas del siglo XXI vienen siendo objeto de análisis en diversos trabajos (Alliaud y Feeney, 2014; Di Franco y Siderac, 2016; Gravino, 2014; Marzoa, Rodríguez y Schoo, 2016; Misuraca y Menghini, 2010; Vior, Misuraca y Más Rocha, 2009). En líneas generales, algunas de estas producciones se centran en aspectos de los PNFD de la primera década, sin constituirlos propiamente en objeto de análisis, así también los trabajos consultados no incorporan aportes de una perspectiva político discursiva, a excepción de algunos autores nacionales (Birgin y Vassiliades, 2018; Morelli e Iturbe, 2018) y de países de la región en los que nos referenciamos y con quienes compartimos aspectos de la analítica y la temática (Lopes, Rodríguez Da Cunha y Costa, 2013; Lopes, 2004; 2005; Dias y Lopes, 2003; Dias, 2004).

Entre 2007 y 2019 se formulan cuatro PNFD. Los tres primeros corresponden al período 20072015 durante las presidencias de Cristina Fernández de Kirchner (Resoluciones CFE N. 23/07, N.ํ 167/12 y N.․188/12) y el cuarto, entre 2015-2019, en la presidencia de Mauricio Macri (Resolución CFE N.․ 286/16). Estos textos, cabe destacar, resultan de procesos articulatorios en el ámbito del CFE, en el que participan los ministros de educación de las provincias argentinas y de la jurisdicción de la Ciudad Autónoma de Buenos Aires juntamente con la instancia nacional, y portan las marcas de los significantes privilegiados que dan sentido a las configuraciones discursivas relacionales, abiertas y precarias (Buenfil Burgos, 2009, p. 117) de las políticas para la formación docente inicial y continua.

Los tres primeros PNFD, de acuerdo con un trabajo previo (Autor y Tourn, 2020), llevan las marcas del nudo significante de la reconstrucción de una identidad para la formación docente, articulada como sistema, vinculada a la educación superior y afirmada diferencialmente con respecto a las configuraciones de las dos últimas décadas del siglo XX. Las condiciones de producción se vinculan, en parte, a un entramado de textos generados en el ámbito del CFE ${ }^{3}$, la Ley de Financiamiento Educativo N N. 26075 (2005) y la Ley de Educación Nacional (LEN) N.ㅇ 26206 (2006), en cuyo marco se crea el Instituto Nacional de Formación Docente (INFD).

En el PNFD 2007-2010 se señala el sentido, orientado a “... consolidar una institucionalidad diferente, potente y dinámica, capaz de reconfigurar en escenarios de mediano y largo plazo, procesos de organización, integración, articulación, fortalecimiento y mejora del sistema formador, sus instituciones y sus vínculos" (Resolución CFE N.ㅇ 23, 2007, p.6) ${ }^{4}$. Además, en este primer texto se patentizan marcas que remiten al Estudio de la calidad y cantidad de oferta de la formación docente, investigación y capacitación en la Argentina ${ }^{5}$-Estudio- (Davini, 2015). Sobre la base del mismo, se organiza el PNFD en torno a diez problemas de la formación docente con sus correspondientes estrategias, objetivos y resultados para el corto y mediano plazo, a implementarse en el período 2007-2010, en áreas de desarrollo prioritarias centradas en lo institucional, lo curricular y la formación continua.

La reconstrucción de una nueva identidad para la formación docente parte del reconocimiento de una ausencia sedimentada en los procesos de la historia educativa reciente. En correspondencia con el

\footnotetext{
${ }^{1}$ El referente empírico, siguiendo a Buenfil Burgos (2012), involucra tanto el proceso que se analiza como las condiciones de producción, y se articula metodológicamente en la construcción del objeto de estudio junto con la dimensión teórica y los interrogantes del investigador.

${ }^{2}$ En este ejercicio recogemos los aportes de Verón, en lo que respecta al análisis en producción, teniendo en cuenta las compatibilidades destacadas por Fair (2008) entre esta analítica y la Teoría de Discurso de Laclau y Mouffe.

${ }^{3}$ En los textos se destacan asimismo las marcas de acuerdos aprobados previamente por las Resoluciones CFE N.. 223/04, N. 241/05, N. 251/05 y la Declaración de diciembre de 2003 "Educación en la Democracia. Balance y Perspectivas", entre otros.

${ }^{4}$ El punto nodal de la reconstrucción de una identidad para el sistema formador que organizó el discurso, se fortalece en definiciones posteriores (Resoluciones CFE N.ㄴ 46/08, N. 98/10 y N. 101/10) y siguientes PNFD para el período 20122015 (Resoluciones CFE N.167/12 y N.188/12), agregándose elementos ligados a la evaluación integral y la incorporación de las tecnologías de la información y la comunicación en la formación docente inicial.

${ }^{5}$ Este estudio recupera algunas de las líneas principales del informe final (Resolución CFE N. 251/05) de la Comisión Federal para la Formación docente (Resolución CFE N. 241/05).
} 
Estudio (Davini, 2015, p.61-64), se señala que esta ausencia se vincula, por un lado, a la dependencia de las instituciones formadoras junto con las de nivel secundario de la Dirección Nacional de Enseñanza Media y Superior (DNEMS) hasta mediados de la década de los ochenta. Por otro, la reconoce como un efecto de la inexistencia de condiciones en el proceso de transferencia de las instituciones de formación docente desde la jurisdicción nacional a las provincias a partir de 1992. Asimismo, la carencia identitaria es entendida como producto de cierta disyunción entre las definiciones de las políticas articuladas para el nivel superior no universitario en el marco de la Ley Federal de Educación (LFE) N.․ 24195 (1993) que lo ligan a lo escolar y, al mismo tiempo, de acuerdo con la Ley de Educación Superior N. ㅇ 24521 (1995), con las particularidades de este nivel. Finalmente, se destaca el grado de fragmentación en los planes de estudio, por efecto de la institucionalización de los contenidos básicos comunes de la formación docente en el marco de la LFE, a partir de los cuales cada provincia llevó a cabo diferentes estrategias para su definición.

En estas opciones reconocemos un aspecto de la dimensión constitutiva de lo político (Mouffe, 2011); esto es, la afirmación de una identidad para la formación docente que excluye la construida en los procesos históricos recientes, fundamentalmente en los años ochenta y noventa. Lo cual involucra, en el terreno de la indecidibilidad, en los términos derridianos (Derrida, 1994), un conjunto de afirmaciones diferenciales que procuran dejar fuera elementos de la secundarización y la fragmentación curricular de la formación docente. Teniendo en cuenta el conjunto de las diez estrategias y objetivos definidos para el corto y mediano plazo en el PNFD, la identidad se configura en torno a una fijación que incluye notas de la institucionalidad universitaria, aunque reconociendo que la misma no agota las posibilidades de la educación superior (Resolución CFE N.ำ 23/07, p.7).

Las condiciones de posibilidad para la reconstrucción de una identidad para la formación docente se asocian, en primer término, con un desarrollo institucional sobre la base de la planificación del sistema y las opciones de formación, un conjunto normativo específico, nuevas dinámicas en la gestión, el gobierno y la organización del sistema en general y las instituciones formadoras en particular, y la formulación de políticas estudiantiles que colaboren con la elección, ingreso y permanencia de estudiantes con buen rendimiento académico ${ }^{6}$. En segundo término, un entramado de definiciones curriculares, articulado a nivel nacional, con desarrollos y evaluaciones en los ámbitos jurisdiccionales e institucionales y, finalmente, la resignificación de las funciones de investigación y experimentación pedagógica, y la formación docente continua (Resolución CFE N.․․ 23/07, p.9).

EI PNFD correspondiente al gobierno de la Alianza Cambiemos, por su parte, da cuenta de una organización en torno al sentido de las competencias profesionales en el currículo, suplementando, mediante una operación de traducción (Lopes, Rodriguez Da Cunha y Costa, 2013), las definiciones establecidas en los Lineamientos Curriculares Nacionales para la Formación Docente Inicial -LCN-, en lo que respecta a la docencia como práctica centrada en la enseñanza (Resolución CFE N.․ 24/07, p.9). En líneas generales, durante el gobierno de la Alianza Cambiemos, se hacen evidentes, tanto en los acuerdos logrados en el ámbito del CFE como en documentos producidos desde el Ministerio de Educación Nacional (MEN), huellas de operaciones discursivas de países hegemónicos a través de agencias internacionales (Filmus, 2017; Puiggrós, 2017).

En el PNFD definido para el período 2016-2021 (Resolución CFE N. 286/16, p.4-5) se expresan las políticas de formación docente, organizadas alrededor de los nudos de sentido justicia educativa, valoración de los docentes, centralidad de la práctica y renovación de la enseñanza. A partir de los mismos se ordenan los objetivos y acciones, centrados en garantizar la cantidad necesaria de docentes con la titulación requerida, y el mejoramiento de la calidad de la formación inicial y de las prácticas de los equipos directivos y docentes. Todos ellos remiten al desarrollo y el fortalecimiento de las competencias profesionales en la formación inicial de los docentes. Es preciso destacar que si bien en el texto del documento se realiza la opción por el significado capacidades, procurando una diferenciación respecto al de competencias, comprendemos que el sentido diseminado refiere al discurso de las competencias en el currículo y que tal uso, en el sentido dado por Laclau (1993), resulta comprensible desde unas condiciones discursivas de posibilidad que en este trabajo asociamos, juntamente con Feldfeber, Puiggrós, Robertson y Duhalde (2018); Filmus (2017), y Saforcada y Feldfeber (2019), a una regularidad neoliberal en las políticas del gobierno de la Alianza Cambiemos. Por otra parte, siguiendo a Lopes (2004, p. 110), cabe advertir la presencia de marcas de operaciones discursivas producidas desde agencias internacionales que reducen las políticas educativas a lo curricular.

Las competencias, por otro lado, no sólo constituyen el punto nodal en la articulación del discurso de las políticas para la formación docente inicial, sino que se diseminan en las definidas para los distintos niveles del sistema educativo nacional, tal como se expresa en las superficies de diversos textos resolutivos y documentales: Declaración de Purmamarca (16/2/16), Red Federal para la Mejora de los Aprendizajes (Resolución CFE N. 284/16), Plan Estratégico Nacional 2016-2021 Argentina Enseña y Aprende (Resolución CFE N.․ 285/16), Lineamientos federales del desarrollo profesional en gestión educativa para equipos directivos y supervisores de los niveles de educación obligatoria (Resolución CFE N.․338/18) y

\footnotetext{
6 Esta opción, de acuerdo a nuestro análisis, constituye una marca que remite a huellas de operaciones discursivas producidas desde las posiciones de agencias internacionales, las cuales, en diferentes documentos (Bruns y Luque, 2014; OCDE, 2009; PREAL, 2004), vienen planteando la necesidad de atraer y "reclutar" a los mejores para la docencia.
} 
Marco Nacional de Integración de los Aprendizajes: hacia el desarrollo de capacidades de la Secretaría de Innovación y Calidad Educativa del MEN (s.f.) ${ }^{7}$.

\section{De las competencias a la fabricación de resultados}

Las competencias como significante maestro (Torfing, 1998) en el momento curricular de la formación docente viene constituyendo un tema de debate en diferentes trabajos (Dias y Lopes, 2003; Dias, 2004; Macedo, 2000), dada su vinculación con las teorías de la pedagogía por objetivos, de la eficiencia social y de transposición de las reglas del mercado a la educación (Cascante Fernández, 2004; Cruz Pineda, 2019; Cullen, 2009; Martins et al., 2013). El carácter privilegiado de esta fijación, teniendo en cuenta las marcas que hemos identificado en fuentes documentales y normativas, da cuenta de la diseminación de los discursos de mejora de la eficiencia y de competencias docentes. Estas configuraciones vienen entramándose desde las últimas décadas del siglo XX en países hegemónicos del ámbito anglosajón (Dias, 2004), estableciéndose como tendencias dominantes en los inicios del siglo XXI a través de una variada producción desde diferentes agencias internacionales (Bruns y Luque, 2014; Diálogo InteramericanoOrganización de Estados Iberoamericanos, 2018; UNESCO/OREALC, 2006; 2018; Vaillant, 2002).

Algunos indicios de estas operaciones discursivas se advierten en las múltiples y convergentes experiencias de países de la región como Brasil (Ministério de Educação e do Desporto, 2001), Chile (Ministerio de Educación, 2008), Perú (Ministerio de Educación, 2012), entre otros, en las que se reconocen algunas trazas de desarrollos desde la América del Norte (Danielson, 2011; Darling-Hammond, 2001). Del mismo modo, el Proceso de Bolonia para la construcción del Espacio Europeo de Educación Superior (Gimeno Sacristán, 2001) y su derivación en el Proyecto Tuning para Latinoamérica (Beneitone et al., 2007), comparten entre sus principales líneas de acción el establecimiento de competencias genéricas y específicas en diferentes campos de la formación universitaria.

En nuestro país, particularmente en los años noventa y comienzos de los dos mil, el discurso de las competencias constituye una marca en algunos textos (Braslavsky, 1999; MEN, 2003). En las políticas nacionales del período analizado se ha diseminado, reactivando elementos presentes en textos documentales inscriptos en los PNFD 2007-2015 (Feldman, 2008; Fleitas et al., 2015), traduciendo los LCN desde la significante competencia.

EI PNFD definido para el período 2016-2019 se organiza, por un lado, en torno al nudo de sentido que instrumentaliza la formación docente con vistas a garantizar las competencias en la educación escolar obligatoria (Resolución CFE N.․ 286/16, p. 3). Por otro lado, la configuración resultante, aglutinada en torno a la mejora de la formación docente desde el significante maestro (Torfing, 1998) competencias profesionales, procura excluir, siguiendo a Cascante Fernández (2004), no sólo el discurso academicista sino también perspectivas de formación reflexivas que resultan antagónicas a las condiciones de posibilidad neoliberales. La operación discursiva se orienta hacia la necesidad de fijar marcos referenciales sobre los conocimientos, capacidades y actitudes en los egresados (Resolución CFE N.ำ 286/16, p.8).

En estas opciones se patentizan marcas de desarrollos recientes en los Estados Unidos (Danielson, 2011), en los que se enfatiza la estrecha relación entre calidad de la formación docente inicial y calidad de los aprendizajes en los niveles de la escolaridad obligatoria. Asimismo, resultan reconocibles las marcas de "buen docente" y "buena enseñanza", las cuales constituyen una regularidad (Foucault, 2002) en las propuestas de países de la región como Chile, sobre la base de los trabajos de Danielson (2011) y Darling-Hammond (2012). Tal como señalan algunas autoras (Dias y Lopes, 2003), se produce una equivalencia entre currículo como desarrollo de competencias, con mejora de la calidad de la formación docente inicial y mejora de los aprendizajes en la escolaridad obligatoria.

En esta línea se articula el Marco Referencial de Capacidades Profesionales de la Formación Docente Inicial -Marco Referencial- (Resolución CFE N․ 337/18), el cual es planteado como una herramienta de política curricular orientado a complementar y enriquecer los LCN, los diseños curriculares de las provincias y los dispositivos nacionales de evaluación curricular (Resolución CFE N.․․ 337/18, p.2). La inclusión del discurso de las competencias en el currículo parte de la construcción de problemáticas con relación a una formulación heterogénea del perfil del egresado en los diseños curriculares provinciales, debilidades en la gestión pedagógica de las instituciones formadoras a partir de una lectura de los resultados de los dispositivos nacionales de evaluación curricular ${ }^{8}$ y, finalmente, dificultades en la definición y organización de las acciones en el campo de la práctica profesional docente (Resolución CFE N ․ㅜ 337/18, p.9-10).

La opción por el significado capacidad, tal como señalamos precedentemente, procura evitar la equivalencia con el de competencia, cuyas condiciones de producción llevan las marcas del mundo del trabajo. En este sentido, resulta comprensible la articulación de un proceso de consulta desde el INFD, a través del cual se precisa un significado de capacidad en términos de:

(...) construcciones complejas de saberes y formas de acción que permiten intervenir en las situaciones educativas (además de comprenderlas, interpretarlas o situarlas) de una manera adecuada y eficaz, para resolver problemas característicos de la docencia. Están asociadas con

\footnotetext{
${ }^{7}$ En línea con los marcos normativos y documentales atravesados por el sentido de las competencias, cabe destacarse el proyecto de ley denominado Plan Maestr@ presentado en 2017, el cual no prosperó en el Congreso Nacional frente al conjunto de críticas formuladas desde distintos sectores.

${ }^{8}$ Estos dispositivos tuvieron lugar en el marco de las Resoluciones CFE N. $167 / 12$ y N. $188 / 12$.
} 
ciertas funciones y tareas propias de la actividad docente orientadas fundamentalmente a enseñar y generar ambientes favorables de aprendizaje, tanto a través de acciones individuales, como de la participación en equipos institucionales del sistema educativo. (Resolución CFE N.ํ337/18, p.1)

En el documento se definen, en primer término, unas competencias generales ligadas al dominio de los saberes a enseñar, la actuación de acuerdo con las características y modalidades del aprendizaje de los alumnos, la gestión de la enseñanza, la intervención en la dinámica grupal, la organización del trabajo escolar en el ámbito institucional y comunitario y, finalmente, el compromiso con la propia formación. En segundo término, a partir de éstas se desagregan un total de treinta capacidades específicas (Resolución CFE N. $337 / 18$, pp. 3-8).

La definición de un Marco Referencial que organiza el currículo sobre la base de competencias, se inscribe en una tendencia hacia la estandarización de la formación docente inicial, tal como ocurre en países de la región, siguiendo los desarrollos de Danielson (2011). Asimismo, las competencias definidas constituyen una condición de posibilidad para la articulación de un dispositivo de fabricación de resultados, orientado hacia la producción de la denominada evidencia; procurando dar cuenta, por un lado, de una ineficiencia de la formación docente inicial actual y, por otro, de la mejora asociada con la institucionalización de un sistema de competencias docentes. A la vez, a partir de los productos de este dispositivo podemos reconocer las trazas de un modelo de identificación (Buenfil Burgos, 2009) desde el que se interpela a funcionarios, equipos técnicos, directivos y docentes, y cuya efectividad radicaría en la reconfiguración de estas posiciones al interior de un discurso eficientista de la formación docente inicial. Siguiendo a Buenfil Burgos, interpelar es:

(...) el acto mediante el cual se nombra a un sujeto, es decir, es la operación discursiva (...) mediante la cual se propone un modelo de identificación a los agentes sociales a los cuales se pretende invitar a constituirse en sujetos de un discurso. Puede ser analizada como una propuesta vertida en distintas modalidades discursivas (lingüística, gestual, icónica, arquitectónica, de vestimenta, etc.) y como unidad significativa de mayor o menor complejidad desde un enunciado simple o una combinación de imagen y enunciados, hasta una configuración extensiva de enunciados (...) (Buenfil Burgos, 2009, p. 92).

En el entramado del dispositivo, tanto en su sentido de productor de resultados como modelo de identificación, se observa cierto "aire de familia" (Wittgenstein, 1988) con el contenido del Informe McKinsey (Barber y Mourshed, 2008). En dicho informe, a partir del análisis de los desempeños de veinte sistemas educativos en evaluaciones internacionales, se establecen etapas para un desarrollo educativo y se identifican seis intervenciones comunes en todos los trayectos de mejora: revisión del plan de estudios y de estándares, recompensas y remuneraciones para docentes y directores, desarrollo de capacidades técnicas de los docentes y directores, evaluación de los estudiantes, establecimiento de sistemas de datos y la publicación de documentos de políticas. Finalmente, en el Informe, se identifican como eventos propicios para comenzar una reforma educativa exitosa una crisis política o económica, informes críticos y de alto nivel sobre el desempeño del sistema y la emergencia de un nuevo líder político o estratégico.

En tal sentido, advertimos huellas del reporte McKinsey en algunas de las modalidades discursivas producidas desde el dispositivo de fabricación de resultados, mediante las cuales se interpela a funcionarios, equipos técnicos, directivos y docentes, fundamentalmente a través de la producción de informes críticos sobre la formación docente inicial. Entre estas modalidades, destacamos la institucionalización de instancias y dispositivos de evaluación de la formación docente; realización de estudios nacionales encomendados a expertos y agencias internacionales para la producción de la denominada evidencia; acciones de formación destinadas a directivos y docentes de los institutos superiores; publicación de compilaciones sobre experiencias "inspiradoras" para el desarrollo de las competencias profesionales; ciclos de conferencias con la participación de expertos ligados mayoritariamente a agencias internacionales y, finalmente, becas de formación en universidades extranjeras.

De ese entramado, destacamos en primer término la instauración de la Secretaría de Evaluación Educativa -SEE- (Poder Ejecutivo Nacional, Decreto N.․ 552/16) y la creación de la Comisión Nacional de Evaluación y Acreditación de la Calidad (CNEAC) de la formación docente (Resolución CFE N.ํ347/18). En el marco de los objetivos asignados a la SEE, se articula el Operativo Enseñar (Resolución CFE N.ำ $324 / 17$ ), orientado a la evaluación de los estudiantes del último año de los profesorados de educación primaria, inicial y secundaria en las disciplinas que forman parte de la estructura curricular del nivel. El dispositivo reactivó y tradujo, en el sentido derridiano (Lopes, Rodriguez Da Cunha y Costa, 2013), elementos de la evaluación integral de la formación docente (INFD, 2014) instaurada a partir de los PNFD 2011 y 2012, e incorporó el modelo teórico de Darling-Hammond (2011), adaptado por Martínez Rizo (2011) para los países latinoamericanos. En línea con la fabricación de resultados, el operativo se orientó a producir información para diagnosticar la formación docente inicial en lo que respecta a competencias de la comunicación escrita (lectura y escritura), la planificación y estrategias de la enseñanza, y evaluación de los aprendizajes (SEE, 2017, pp. 8, 11-12).

Los resultados del operativo, si bien resultan orientativos, convergen hacia el punto discursivo de las competencias, como así también hacia los nudos de sentido que aglutinan la política de formación docente expresada en el PNFD. En tal sentido, se señala, entre otros aspectos, la diversidad y 
heterogeneidad de la formación inicial y las instituciones del sistema formador; la relevancia de la práctica de la enseñanza en la formación inicial, teniendo en cuenta los óptimos desempeños obtenidos por aquellos estudiantes que cuentan con un título docente previo; la correlación entre mejores resultados en el operativo con el criterio de elección de la carrera docente, con las condiciones de infraestructura y equipamiento de los establecimientos, con la antigüedad y titularidad de los directivos de los institutos, con el tamaño medio de las instituciones y con la formación para un nivel de escolaridad en particular (SEE, 2018a, pp. 217-221).

En este mismo sentido, desde la SEE se implementó en 2018 la denominada autoevaluación en institutos de formación docente (SEE, 2018b). Si bien el dispositivo se deriva de las definiciones sobre la evaluación integral de la formación docente comprendidas en los PNFD 2007-2015, la evaluación se resignifica desde el discurso de mejora de la calidad educativa, en línea con la teoría del cambio (SEE, 2018 b, pp. 8). A partir de un significado multidimensional de calidad educativa, se desagregan dimensiones que comprenden el contexto, los procesos académicos, la gestión institucional, el vínculo con la comunidad, el gobierno y el impacto institucional (SEE, 2018b, p.10).

En el entramado del dispositivo de fabricación de resultados, la CNEAC de la formación docente, se instituye como instancia responsable de producir, por una parte, información acerca del cumplimiento de requisitos y condiciones en las instituciones y carreras de formación docente de todo el país, de acuerdo con normativas nacionales y federales y, por otra, emitir los dictámenes correspondientes para el otorgamiento de la validez nacional de los títulos y las carreras. Las posiciones que integran este ámbito están representadas por cada región del país, el INFD, la SEE, la provincia cuyos institutos sean evaluados, académicos de reconocida trayectoria en formación docente propuestos por el MEN y con acuerdo del CFE, y el propio MEN en la coordinación de la CNEAC (Resolución CFE N.․ 347/18). Cabe señalar el parecido de familia (Wittgenstein, 1988) de esta instancia con la Comisión Federal de Evaluación (CoFEv), la cual, desde 2008, tuvo a su cargo el análisis de los diseños curriculares producidos por las provincias, verificando el cumplimiento de los requisitos establecidos por el CFE a través de los LCN para el otorgamiento de la validez de los títulos (Resolución MEN N.․ 2170/08). No obstante, el sentido de la CNEAC sólo resulta comprensible desde las condiciones de posibilidad del dispositivo de fabricación de resultados y, como indicamos previamente, a una regularidad neoliberal de las políticas educativas a fines de la segunda década de los dos mil.

En segundo término, la publicación de documentos resultantes de estudios nacionales encomendados a expertos e investigadores de la educación superior y agencias internacionales con sede en el país, en línea con la producción de evidencia, constituye otra modalidad discursiva que se integra al modelo de identificación desde el que se interpela. Las publicaciones de documentos con resultados, si bien constituyen producciones sustantivas, están orientadas desde el sentido de exponer ciertas ineficiencias del sistema formador para avanzar desde el punto nodal de las competencias.

Este conjunto de documentos se integra por dos estudios nacionales; el primero de ellos enfocado en las distancias entre un deber ser didáctico, las prácticas de enseñanza en el contexto del aula en los niveles primario y secundario, y la reproducción de tradiciones en la formación inicial (SEE-INFD, 2019a, p.5), y un segundo estudio centrado en la organización y el funcionamiento del campo de las prácticas en la formación docente inicial, traccionado desde el significante competencias de acuerdo al Marco Referencial (SEE-INFD, 2019b, pp.144-173; pp.185-186; p.225). Por otra parte, estudios exploratorios sobre modelos organizacionales y pedagógicos de las instituciones de formación docente (Vaillant y Fumagalli, 2018), la formación de directores y supervisores a nivel internacional (IIPE-UNESCO, 2018) y una aproximación a los costos laborales en las carreras de formación docente y técnico profesional (Dirección de Información y Estadística Educativa, 2017). Cabe destacar otras publicaciones incluidas en una serie sobre la investigación en los institutos de formación docente de todo el país, organizadas temáticamente en torno a las prácticas de enseñanza (MEN, 2018a), la formación docente (MEN, 2018b) y la inclusión, trayectorias y diversidad cultural (MEN, 2018c). En fin, la serie de modalidades discursivas producidas se comprenden en relación con los nudos de sentido del PNFD y las problemáticas construidas como condición de posibilidad para la articulación del Marco Referencial.

La formación de directivos y docentes de los institutos de formación docente, en tercer término, constituye otra modalidad discursiva en la cadena de interpelaciones desde el modelo de identificación eficientista. La actualización académica en formación docente (Resolución MEN № 2146/18) se asienta, por un lado, en una correspondencia entre calidad de la enseñanza y calidad del aprendizaje, interpelando las prácticas de enseñanza vigentes, dada su ineficacia probada a través de los resultados que ofrecen las pruebas de evaluación y, por otro, se organiza en torno a las competencias profesionales. En este último sentido, una vez concluida la formación, se convocó a los docentes postitulados a producir propuestas sobre la base de las competencias generales establecidas en el Marco Referencial, cuya compilación forma parte de una publicación (MEN, 2019c).

Finalmente, es preciso destacar la realización de ciclos de conferencias y becas internacionales sobre formación docente. Las dos ediciones de las conferencias, denominadas miradas del mundo sobre la formación docente, en línea con las operaciones de interpelación, reunieron un conjunto de especialistas latinoamericanos, estadounidenses y portugueses que expusieron las políticas de formación docente implementadas en sus países de origen, los resultados y eficacia en la mejora de la enseñanza. Las becas internacionales tuvieron como destino las universidades de Jamk (Finlandia) y de California (Los Ángeles, EEUU) y estuvieron dirigidas a funcionarios, equipos técnicos y directivos de todo el sistema formador del país. 
En último lugar, en línea con la profundización de los distintos aspectos que hemos destacado del modelo de identificación operado a través del dispositivo de fabricación de resultados, consideramos la memoria de las acciones desarrolladas desde el INFD en los últimos cuatro años, cuyo documento avanza en un conjunto de sugerencias para el futuro que refuerzan el sentido de las políticas de formación docente en torno a los significados competencia, estandarización, evaluación, acreditación, eficiencia, entre otros (INFD, 2019, pp.92-95).

\section{Consideraciones finales}

El análisis político de documentos oficiales, nos ha posibilitado reconocer algunos indicios que remiten a una fijación parcial de sentido en torno al punto discursivo privilegiado de las competencias profesionales en el discurso de la política educativa para la formación inicial de docentes en Argentina durante el período 2015-2019. Esta fijación parcial de sentido resulta comprensible desde unas condiciones de posibilidad atravesadas por una regularidad neoliberal de las políticas educativas en general durante el gobierno de la Alianza Cambiemos. Las políticas para la formación docente inicial, en particular, resultan de un ejercicio de traducción desde el significado competencias profesionales de los Lineamientos Curriculares Nacionales instaurados en 2007, en línea con operaciones discursivas articuladas desde países hegemónicos y diseminadas a través de agencias internacionales, cuyas huellas pueden reconocerse en los discursos de países de la región.

La organización curricular de la formación docente inicial, sobre la base del significado competencias profesionales, por otra parte, se constituye en una condición de posibilidad para el entramado de lo que caracterizamos como un dispositivo de fabricación de resultados, orientado hacia la producción de la denominada evidencia. De los productos de este dispositivo, hemos considerado modalidades discursivas documentales y normativas referidas a instancias e informes de evaluación de la formación docente inicial, estudios nacionales, propuestas formativas destinadas a directivos y docentes del sistema formador, entre otros. De acuerdo con nuestro análisis, el sentido del entramado de este dispositivo se orienta, por un lado, a la construcción de un estado deficitario de la formación docente inicial actual y, por otro, hacia la institucionalización de un sistema de competencias docentes como vía de mejora de la eficacia. A la vez, a partir del dispositivo y sus productos podemos reconocer las trazas de un modelo de identificación desde el que se interpela a funcionarios, equipos técnicos, directivos y docentes, y cuya efectividad radicaría en la reconfiguración de estas posiciones al interior de un discurso eficientista de la formación docente inicial, organizado nodalmente en torno a las competencias profesionales como condición de posibilidad para la mejora.

Una última reflexión derivada del análisis realizado en esta producción, confrontada con el ejercicio de la docencia y el gobierno en una institución formadora de docentes, nos permite considerar cierta sedimentación en la constitución de las distintas posiciones y los juegos de lenguaje de las prácticas de formación, no sólo en el sistema formador sino también en los niveles de la escolaridad obligatoria. En este sentido, asistimos a la resignificación del saber académico y escolar en términos de competencias estandarizadas, la investigación educativa asimilada a la producción de evidencia despojada de teoría, las prácticas de evaluación reducidas a esquemas tecno-burocráticos de confección y completamiento de rúbricas, la planificación estratégica y situacional, el liderazgo directivo instructivo, para mencionar algunos efectos de las operaciones de interpelación.

Finalmente, aún no avizoramos en las proximidades de la tercera década de los años dos mil una frontera política a partir de la cual reconfigurar diferencialmente los discursos de las políticas educativas para la formación docente inicial y continua en el sistema educativo argentino, tras las articulaciones durante el gobierno de la Alianza Cambiemos.

Referencias bibliográficas

Alliaud, A. y Feeney, S. (2014). La formación docente en el nivel superior de Argentina: hacia la conformación de un sistema integrado. Revista Latinoamericana de Políticas y Administración de la Educación, № 1, pp.125-134. Recuperado de http://relapae.untref.edu.ar/numero-1/.

Autor y Tourn, L. (2020). Planes de formación docente en Argentina (2007-2019): una lectura desde la dimensión de lo político. Linhas Críticas, 26, pp.1-20. Recuperado de https://periodicos.unb.br/index.php/linhascriticas/article/view/27688/26981

Birgin, A. y Vassiliades, A. (2018). Políticas estudiantiles en la formación docente: problemas pedagógicos y debates en perspectiva Suramericana. Revista Archivos Analíticos de Políticas Educativas, 26(159), pp. 1-15. Recuperado de https://epaa.asu.edu/ojs/article/view/3140/2177.

Braslavsky, C. (1999). Bases, orientaciones y criterios para el diseño de programas de formación de profesores, Revista Iberoamericana de Educación. № 19, pp. 13-50. Recuperado de https://rieoei.org/RIE/article/view/1054.

Buenfil Burgos, R. (1992). Análisis de discurso y educación. México DF: Departamento de Investigaciones Educativas (DIE). Centro de investigación y de estudios avanzados del Instituto Politécnico Nacional. Documento № 26. (2007). Introducción, en Padierna Jiménez, P. y Maríñez, R. (Coord.), Educación y comunicación. Tejidos desde el análisis político de discurso (15-35). México: Casa Juan Pablos - Programa de Análisis Político de Discurso e Investigación (PAPDI).

(2009). Análisis político del discurso e historia de la educación, en: Pini, M. (Comp.), Discurso y educación. Herramientas para el análisis crítico (77-121). San Martín: Universidad Nacional de San Martín Edita.

(2011). Apuntes sobre los usos de la teoría en la investigación social. Consideraciones metodológicas en investigación social. Alemania: Editorial Académica Española.

(2012). La teoría frente a las preguntas y el referente empírico en la investigación. En: Jiménez García, M. (Coord.). Investigación educativa. Huellas metodológicas (51-71). México DF: Juan Pablos Editores Seminario de Análisis del Discurso. 
(2019). Ernesto Laclau y la investigación educativa en Latinoamérica: implicaciones y apropiaciones del análisis político de discurso. Buenos Aires: CLACSO.

Cascante Fernández, C. (2004). La reforma de los planes de estudio. Un análisis político de los discursos sobre la formación inicial de los profesionales de la educación. Revista Interuniversitaria de Formación del Profesorado, 18(3), pp. 145-167. Recuperado de https://www.redalyc.org/pdf/274/27418309.pdf.

Cruz Pineda, O. (2008). Los documentos como textos. Los textos como referentes. Aproximaciones analíticas. En Pineda, O. y Echavarría Canto, L. (Coord.), Investigación social. Herramientas teóricas y análisis político de discurso (128-139). México: Juan Pablos/Programa de Análisis Político de Discurso e Investigación.

(2019). Configuración de discurso pedagógico y reformas educativas en México. Una aproximación a su análisis. Revista Mexicana de Investigación Educativa, 24(81), pp. 565-591. Recuperado de http://www.scielo.org.mx/scielo.php?script=sci arttext\&pid=S1405-6662019000200565.

Cullen, C. (2009). Entrañas éticas de la identidad docente. Buenos Aires: La Crujía Ediciones.

Darling-Hammond, L. (2012). Desarrollo de un enfoque sistémico para evaluar la docencia y fomentar una enseñanza eficaz. Pensamiento Educativo. Revista de Investigación Educacional Latinoamericana, 49(2), pp. 1-20. Recuperado de http://www.pensamientoeducativo.uc.cl/files/journals/2/articles/479/public/479-357-1-PB.pdf.

Derrida, J. (1994). Márgenes de la filosofía. Madrid: Ediciones Cátedra.

Dias, R. y Lopes, A. (2003). Competências na formação de professores no Brasil: o que (não) há de novo. Educação \& Sociedade, 24(85), pp.1155-1171. Recuperado de http://www.scielo.br/pdf/es/v24n85/a04v2485.pdf.

Dias, R. (2004). A recontextualização do conceito de competências no currículo da formação de professores no Brasil. Teias, 5(8-9), pp.1-12. Recuperado de https://www.epublicacoes.ueri.br/index.php/revistateias/article/view/23940/16913.

Di Franco, M.; Di Franco, N. y Siderac, S. (2016). La formación docente en las políticas públicas: el campo de las prácticas como posibilidad. Praxis \& Saber, 7(15), pp.17-40. Recuperado de https://revistas.uptc.edu.co/index.php/praxis saber/issue/view/449.

Fair, H. (2008). Laclau y Verón: discusiones teóricas y contribuciones para la praxis en dos teorías del discurso. Revista Anual de la Unidad de Historiografía e Historia de las Ideas, 9(10), pp.9-24.

Feldfeber, M., et al. (2018). La privatización educativa en Argentina. Buenos Aires: CTERA.

Filmus, D. (2017). (Comp.). Educar para el mercado. Escuela, universidad y ciencia en tiempos de neoliberalismo. Buenos Aires: Octubre Editorial.

Foucault, M. (2002). La arqueología del saber. Buenos Aires: Siglo XXI Editores.

Gimeno Sacristán, J. (Comp.) (2008). Educar por competencias, ¿qué hay de nuevo? Madrid: Ediciones Morata.

Giovine, R. y Suasnábar, J. (2012). Los textos legales como analizador de las políticas educativas: consideraciones teórico - metodológicas. En: Red de Estudios Teóricos y Epistemológicos en Política Educativa, I Jornadas Latinoamericanas de estudios epistemológicos en política educativa, 15-16 de noviembre de 2012. Recuperado de: http://www.relepe.org/images/ponencia\%203\%20giovine\%20suasnabar\%20enviar.pdf

Gravino, M. (2014). Políticas de formación y trabajo docente: un relato sobre la reforma curricular en Neuquén, Argentina (2002-2010). En: Badano, y Ríos (Coord.). Trabajo docente, pensamiento crítico: políticas, prácticas, saberes y transformación social (209-215). Paraná: Editorial Fundación La Hendija

Howarth, D. (2005). Aplicando la teoría del discurso: el método de la articulación. Studia Politicae, № 5, pp. 37-88. Recuperado http://www2.ucc.edu.ar/portalucc/archivos/File/CP_y_RRII/Revista_Studia_Politicae/05/Articulos/Howarth.pdf.

Laclau, E. (1993). Nuevas reflexiones sobre la revolución en nuestro tiempo. Buenos Aires: Nueva Visión. y Mouffe, Ch. (1987). Hegemonía y estrategia socialista. Hacia una radicalización de la democracia. Buenos Aires: Siglo XXI editores.

Lopes, A. (2004). Políticas curriculares: continuidade ou mudança de rumos? Revista Brasileira de Educação, 26, pp.109118.

Recuperado de http://www.scielo.br/scielo.php?script=sci_arttext\&pid=\$14134782004000200009.

Lopes, A. (2005). Política de curriculo: recontextualização e hibridismo. Currículo sem Fronteiras, 5(2), pp.50-64. Recuperado de http://www.curriculosemfronteiras.org/art_v5_n2.htm.

Lopes, A., Rodrigues Da Cunha, E. y Costa, H. (2013). Da recontextualização á tradução: investigando políticas de currículo. Currículo sem Fronteiras, 13(3), pp.392-410. Recuperado de http://www.curriculosemfronteiras.org/vol13iss3articles/lopes-cunha-costa.pdf.

Macedo, E. (2000). Formação de professores e diretrizes curriculares nacionais: para onde caminha a educação? Teias, 1(2), $\quad$ pp.1-16. Recuperado https://www.epublicacoes.uerj.br/index.php/revistateias/article/view/23845/16818.

Martínez Rizo, F. (2016). La evaluación de docentes de educación básica. Una revisión de la experiencia internacional. México: Instituto Nacional para la Evaluación de la Educación.

Martins, I. et. al. (2013). Las competencias en las políticas de currículum de ciencias: los casos de Brasil y Portugal. Revista Mexicana de Investigación Educativa, 18(56), pp.37-62. Recuperado de http://www.scielo.org.mx/pdf/rmie/v18n56/v18n56a3.pdf.

Marzoa, K.; Rodríguez, M. y Schoo, S. (2011). Dos décadas de reformas en las políticas de formación docente de dos jurisdicciones. En Sociedad Argentina de Estudios Comparados en Educación. IV Congreso Nacional/III Encuentro Internacional de Estudios Comparados en Educación ¿Hacia dónde va la educación en la Argentina y en América Latina? Construyendo una nueva agenda, Buenos Aires, 1-21. Recuperado de http://www.saece.com.ar/autores4.php.

Misuraca, M. y Menghini, R. (2010). La formación docente en la Argentina del siglo XXI: ¿Consolidación de las tendencias de los '90? Profesorado. Revista de Curriculum y Formación del Profesorado. 14(2), pp.251-266. Recuperado de https://recyt.fecyt.es/index.php/profesorado/article/view/42917.

Morelli, S. e Iturbe, E. (2018). Tensiones y traducciones en las políticas curriculares para la formación docente en Argentina. Educação Unisinos, 22(1), pp.44-52. Recuperado de http://revistas.unisinos.br/index.php/educacao/article/view/edu.2018.221.05/60746119.

Mouffe, Ch. (2011). En torno a lo político. Buenos Aires: Fondo de Cultura Económica. (Versión original, 2005).

Puiggrós, A. (2017). Adiós, Sarmiento. Educación pública, iglesia y mercado. Buenos Aires: Colihue.

Ruiz Muñoz, M. (2005). Imbricación de lo político y lo pedagógico en los procesos de educación de adultos. Dos estudios de caso. Pátzcuaro, México: Centro de Cooperación Regional para la Educación de Adultos en América Latina y el Caribe. 
Saforcada, F. y Feldfeber, M. (Comp.) (2019). La regulación del trabajo y la formación docente en el siglo XXI: miradas desde Argentina. Buenos Aires: Editorial de la Facultad de Filosofía y Letras, Universidad de Buenos Aires.

Torfing, J. (1998). Un repaso al análisis de discurso. En: R. Buenfil Burgos (Coorda ${ }^{\text {.) }}$, Debates políticos en los márgenes de la modernidad (31-52). México: Seminario Programa de Análisis Político de Discurso - Plaza y Valdés.

Treviño, E. (2018). El estudio de las políticas desde una mirada de lo político. En F. Fuentes Navarro (Coord.), Políticas públicas y politicidad en educación. Configuraciones teóricas e investigativas (58-73). México: Secretaría de Educación de Veracruz.

Verón, E. (1993). La semiosis social. Fragmentos de una teoría de la discursividad. Barcelona: Gedisa. (2004). Fragmentos de un tejido. Barcelona: Gedisa Editorial.

Vior, S.; Misuraca, M. y Más Rocha, S. (comp.) (2009). Formación de docentes. ¿Qué cambió después de los '90 en las políticas, los currículos y las instituciones? Buenos Aires: Jorge Baudino Ediciones.

Wittgenstein, L. (1988). Investigaciones filosóficas. Barcelona: Grupo Editorial Grijalbo.

\section{Documentos}

Argentina. Consejo Federal de Educación (27 de noviembre de 2003). Declaración Educación en la democracia. Balance

y perspectivas. Recuperado de https://cfe.educacion.gob.ar/doc_pdf/educ_y_demo.pdf.

(12 de febrero de 2016). Declaración de Purmamarca. Recuperado de
https://www.argentina.gob.ar/sites/default/files/declaracion-de-purmamarca.pdf.

INFD (2014). Evaluación integral de la formación docente. Evaluación de estudiantes, etapa 2014. Documento base. Recuperado de ontent/uploads/2020/01/1_ev estudiantes docu base v228 1.pdf

Ministerio de Educación. Secretaría de Educación Subsecretaría de Equidad y Calidad. Dirección Nacional de Gestión Curricular y Formación Docente (2003). Formación y transferencias de saberes y prácticas docentes para la inclusión educativa social. Competencias profesionales en la formación de docentes. Recuperado de http://www.bnm.me.gov.ar/giga1/documentos/EL003907.pdf.

Ministerio de Educación. Dirección de Información y Estadística Educativa (2017). Las carreras de formación docente y técnico profesionales: una aproximación a sus costos laborales. Buenos Aires.

Ministerio de Educación. Secretaría de Evaluación Educativa. (2017). Enseñar. Evaluación diagnóstica de estudiantes del último año de formación docente en Argentina. Documento Marco. Recuperado de https://www.argentina.gob.ar/sites/default/files/documento_marco_ensenar_0.pdf

Ministerio de Educación. Secretaría de Evaluación Educativa. (2018a). Evaluación diagnóstica 2017. Estudiantes avanzados de carreras docentes. Recuperado de https://www.argentina.gob.ar/sites/default/files/reporte_nacional_ensenal_2017.pdf.

Ministerio de Educación. Secretaría de Evaluación Educativa. (2018b). Autoevaluación en institutos de formación docente. Documento marco.

Recuperado

https://www.argentina.gob.ar/sites/default/files/documento marco autoevaluacion.pdf.

Ministerio de Educación. INFD. (2018a). Prácticas docentes y de enseñanza. Recuperado de https://www.educ.ar/sitios/educar/resources/150626/practicas-docentes-y-de-enseanza/download.

Ministerio de Educación. INFD. (2018b). Formación docente. Recuperado de http://repositorio.educacion.gov.ar:8080/dspace/bitstream/handle/123456789/110476/volumen_2_formacion_ docente.pdf?sequence $=1$.

Ministerio de Educación. INFD. (2018c). Inclusión, trayectorias y diversidad cultural. Recuperado de http://repositorio.educacion.gov.ar/dspace/bitstream/handle/123456789/110477/volumen_3_inclusion.pdf?seq uence $=1$.

Secretaría de Innovación y Calidad Educativa - INFD. (2019a). Estudio nacional 2016-2017. Prácticas de enseñanza en el contexto del aula en los niveles primario y secundario. Recuperado de http://dgescba.edu.ar/bd_dges/recursos/noticias_destacadas/2019/2019_12_13_10_31_24_INFoDprimer_Estudio_Nacional_2016-2017.pdf.

Secretaría de Inñovación y Calidad Educativa - INFD. (2019b). Estudio nacional 2017-2018. El campo de las prácticas en la formación docente inicial. Organización, funcionamiento y tensiones. Recuperado de http://dgescba.edu.ar/bd dges/recursos/noticias destacadas/2019/2019_12_13_10_27_17_INFoDSegundo_Estudio_Nacional_2017-2018_A.pdf.

Secretaría de Innovación y Calidad Educativa - INFD. (2019c). De formadores para formadores. Experiencias para el desarrollo de capacidades profesionales en la formación docente inicial. Recuperado de https://drive.google.com/file/d/1aja76KUcFQI6Cv7b7Wd5SrieC199kpXY/view.

INFD. (2019). Memoria del Instituto Nacional de Formación Docente 2016-2019. Renovar la enseñanza, garantizar los aprendizajes. Recuperado de https://www.educ.ar/sitios/educar/resources/150828/memoria-del-institutonacional-de-formacion-docente-2016-2019/download.

Secretaría de Innovación y Calidad Educativa (s/f). Marco Nacional de Integración de los Aprendizajes: hacia el desarrollo de capacidades. Recuperado de http://www.mendoza.edu.ar/wp-content/uploads/2017/03/Capacidades.pdf.

Poder Ejecutivo Nacional (2017). Proyecto de Ley Plan Maestr@. Recuperado de http://www.bnm.me.gov.ar/giga1/documentos/EL005527.pdf.

Barber, M. y Mourshed, M. (2008). Cómo hicieron los sistemas educativos con mejor desempeño del mundo para alcanzar sus objetivos. Santiago de Chile: Editorial San Marino.

Beneitone, P., Esquetini, C., González, J.; Marty Maletá, M., Siufi, G. y Wagenaar, R. (Ed.) (2007). Reflexiones y perspectivas de la educación superior en América Latina. Informe final -Proyecto Tuning- América Latina 20042007. Bilbao: Publicaciones de la Universidad de Deusto. Recuperado de http://tuningacademy.org/wpcontent/uploads/2014/02/TuningLAllI_Final-Report_SP.pdf

Bruns, B. y Luque, J. (2014). Profesores excelentes. Cómo mejorar el aprendizaje en América Latina y el Caribe. Washington DC: Banco Mundial. https://openknowledge.worldbank.org/bitstream/handle/10986/20488/Spanish-excellent-teachers-report.pdf.

Chile, Ministerio de Educación (2008). Marco para la buena enseñanza. Santiago de Chile: Impresora Maval. Recuperado de https://www.cpeip.cl/marco-buena-ensenanza/.

Danielson, Ch. (2011). Competencias docentes: desarrollo, apoyo y evaluación. En Serie Documentos Programa de Promoción de la Reforma Educativa en América Latina y el Caribe-PREAL-, 51. Chile: PREAL. Recuperado 
de

http://www.empresariosporlaeducacion.org/sites/default/files/3_doc51_competenciasdocentes_enero2011.pdf

Davini, M. (2015). Estudio de la calidad y cantidad de oferta de la formación docente, investigación y capacitación en la Argentina: informe final. Buenos Aires: Ministerio de Educación de la Nación.

Diálogo Interamericano y Organización de Estados Iberoamericanos (2018). Argentina: el estado de las políticas públicas docentes. Informe de seguimiento PREAL. Buenos Aires. Recuperado de http://panorama.oei.org.ar/_dev2/wpcontent/uploads/2018/03/ARGENTINA-El-estado-de-las-pol\%C3\%ADticas-p\%C3\%BAblicas-docentes.pdf.

Feldman, D. (2008). Treinta y seis capacidades para la actividad docente en escuelas de educación básica. Material de trabajo. Dirección Nacional de Formación e Investigación. Área de Desarrollo Curricular. INFD. Recuperado de https://isfdef-for.infd.edu.ar/sitio/documentaciones/upload/Feldman2c_Treinta_y_seis_capacidades_1.pdf.

Fleitas, D., Dente, L., Marchegiani, M., Michel, S., Pico, L., Rodríguez, C., Sartirana, L., Senatore, A. y Toranzos, L. (2015). Evaluación integral de la formación docente. Evaluación de estudiantes 2013-2015. Memoria técnica. Ciudad Autónoma de Buenos Aires: Ministerio de Educación. Recuperado de https://cedoc.infd.edu.ar/upload/Ev_estudiantes_Memoria_tecnica_sept2015_1.pdf.

IIPE-UNESCO Buenos Aires (2018). Estudio exploratorio sobre modelos internacionales de formación de directores y supervisores: un análisis en clave comparada. Informe final. Recuperado de https://panorama.oei.org.ar/_dev2/wp-content/uploads/2018/10/Informe-final-Gonzalo-Mu\%C3\%B1oz.pdf.

OCDE (2009). Los docentes son importantes: atraer, formar y conservar a los docentes eficientes. París: OCDE publicaciones.

PREAL (2004). Maestros en América Latina: nuevas perspectivas sobre su formación y desempeño. Santiago de Chile.

República Federativa del Brasil. Ministério de Educação e do Desporto. Conselho Nacional de Educação. (2001) Diretrizes Curriculares Nacionais para a Formação de Professores da Educação Básica, em nível superior, curso de licenciatura, de graduação plena. Brasília, DF: MEC/CNE. Recuperado de http://portal.mec.gov.br/pet/323-secretarias-112877938/orgaos-vinculados-82187207/12861-formacaosuperior-para-a- docencia-na-educacao-basica.

UNESCO/OREALC (2006). Modelos innovadores en la formación inicial docente. Estudios de casos de modelos innovadores en la formación docente en América Latina y Europa. Santiago de Chile: UNESCO/OREALC. Recuperado de https://unesdoc.unesco.org/ark:/48223/pf0000146544.

(2018). Formación inicial docente en competencias para el siglo XXI y pedagogías para la inclusión en América Latina. Análisis comparativo de siete casos nacionales. Santiago de Chile: UNESCO/OREALC. Recuperado de: https://www.unisantos.br/wp-content/uploads/2018/09/INFORME-REGIONAL-SXXI-aINCLUSION-version-FINAL-JUNHO2018.pdf

Vaillant, D. (2002). Formación de formadores. Estado de la práctica. Serie Documentos Programa de Promoción de la Reforma Educativa en América Latina, 25. Santiago de Chile: Editorial San Marino. Recuperado de https://www.fundacionluminis.org.ar/listado-orientativo/formacion-de-formadores-estado-de-la-practicacuadernillo-25.

y Fumagalli, L. (2018). Estudio exploratorio sobre modelos organizacionales y pedagógicos de instituciones dedicadas a la formación docente inicial. Un análisis en clave comparada. Informe final. Realizado a solicitud del Instituto Nacional de Formación Docente de la República Argentina por IIPE-UNESCO. Recuperado de http://www.denisevaillant.com/wp-content/uploads/2019/03/Informe-final-Estudio-formaci\%C3\%B3n-docenteINFOD-abril-2018-Vaillant-v4-1.pdf

\section{Normativas}

Argentina, Senado y Cámara de Diputados de la Nación (14 de abril de 1993). Ley Federal de Educación. Buenos Aires: Boletín Oficial, 5 de mayo de 1993.

(20 de julio de 1995). Ley de Educación Superior. Buenos Aires: Boletín Oficial, 10 de agosto de 1995

(21 de diciembre de 2005). Ley de Financiamiento Educativo. Buenos Aires: Boletín Oficial, 12 de enero de 2006.

(14 de diciembre de 2006). Ley de Educación Nacional. Buenos Aires: Boletín Oficial, 28 de diciembre de 2006. 2016.

Argentina, Consejo Federal de Educación (11 de agosto de 2004). Resolución N. 223. Buenos Aires. (15 de junio de 2005). Resolución N.․ 241. Buenos Aires.

(11 de agosto de 2005). Resolución N. - 251. Buenos Aires. (7 de noviembre de 2007). Resolución N. 23. Buenos Aires. (7 de noviembre de 2007). Resolución N.24. Buenos Aires. (29 de mayo de 2008). Resolución N. ⒍ Buenos Aires. (12 de mayo de 2010). Resolución N.9 98. Buenos Aires. (24 de junio de 2010). Resolución N ․ 101. Buenos Aires. (28 de marzo de 2012). Resolución N. 167. Buenos Aires. (5 de diciembre de 2012). Resolución N.188. Buenos Aires. (29 de junio de 2016). Resolución N. 284. Tucumán.

(23 de agosto de 2016). Resolución N. 285. San Luis. (23 de agosto de 2016). Resolución N. 286. San Luis. (2 de agosto de 2017). Resolución N. -324 . Santiago del Estero.

(5 de abril de 2018). Resolución N. 337. Buenos Aires.

(5 de abril de 2018). Resolución N. 338 . Buenos Aires.

(19 de diciembre de 2018). Resolución N. 347. Buenos Aires.

Ministerio de Educación (29 de diciembre de 2008). Resolución N. 2170. Buenos Aires.

Ministerio de Educación (6 de agosto de 2018). Resolución N. 2146. Buenos Aires.

Perú. Ministerio de Educación. (27 de diciembre de 2012). Resolución N.o 0547. Lima. 